\title{
Resistive magnetohydrodynamic modeling for 3D plasmas
}

\author{
Robin Storer $^{\mathrm{a}, *}$, Henry Gardner ${ }^{\mathrm{b}}$ \\ a School of Chemistry, Physics and Earth Sciences, The Flinders University of South Australia GPO Box 2100, Adelaide 5001, Australia \\ ${ }^{\mathrm{b}}$ Department Computer Science, FEIT Australian National University, Canberra ACT 0200, Australia
}

\begin{abstract}
This paper reports on the development of a three-dimensional resistive magnetohydrodynamic code for stellarator-like plasmas, SPECTOR-3D. The code is designed to find the characteristic modes of the linear perturbations away from the plasma equilibrium, with an emphasis on the unstable modes. @ 2001 Elsevier Science B.V. All rights reserved.
\end{abstract}

PACS: 52.30.Jb; 52.55.Hc; 52.65.-y; 52.35.Py

Keywords: Magnetohydrodynamics; Resistive instabilities; Stellarator plasmas; Computational modeling

\section{Introduction}

The study of the dynamics of plasmas has been considerably progressed by advanced computational modeling which can determine, for example, the magnetic configurations which can lead to a stable plasma. Typical stability codes rely on numerically solving the linearized magnetohydrodynamic (MHD) equations and they have been very successful in predicting the behaviour of plasmas in tokamaks. A number of codes have been developed for resistive MHD applied to axisymmetric toroidal plasmas (FAR [1], MARS [2], CASTOR [3], ARES [4,5] and SPECTOR [6]) and a modification of FAR has been developed for stellarators [7]. Stellarators offer the possibility of steady state operation but have the added complication of three-dimensional helical field structures. Three dimensional ideal MHD codes such as TERPSICHORE [8] and CAS3D [9] have been developed to study the stability of stellarator configurations, however resistivity has the potential to alter the topography of the fields and can give rise to 'tearing' modes which tear apart the magnetic fields.

This paper reports on the development of a three-dimensional resistive magnetohydrodynamic code for stellarator-like plasmas, SPECTOR-3D. The code is designed to find the characteristic modes of the linear perturbations away from the plasma equilibrium, with an emphasis on the unstable modes. The characteristic unstable modes need to be described in terms of a full double Fourier expansion. In addition the 3D algebraic analysis is significantly more complex. The 3D plasma equilibrium code, VMEC [10], was interfaced as part of the input to SPECTOR-3D and a mapping to a new coordinate system - Boozer coordinates — was used from the code TERPSICHORE. Boozer coordinates are a non-orthogonal set of coordinates and so there are

\footnotetext{
* Corresponding author.

E-mail address: robin.storer@flinders.edu.au (R. Storer).
} 
considerable algebraic complexities in reducing the vector MHD equations using these coordinates. We developed a Mathematica program which carried out all of the non-orthogonal vector calculus, incorporating the double Fourier transform reduction, and as a bonus formulated the terms directly as FORTRAN statements which were incorporated into the code, thus minimizing errors. The reduction of the equations was done in terms of the 6 components of the vector potentials of the perturbed magnetic field and the velocity, which were in turn Fourier transformed in both the toroidal and poloidal angles.

\section{Basic equations}

The equations used are the incompressible set of linearized resistive MHD equations (with appropriate normalization):

$$
\begin{aligned}
& \frac{\partial \mathbf{v}}{\partial t}=(\nabla \times \mathbf{B}) \times \mathbf{b}+(\nabla \times \mathbf{b}) \times \mathbf{B}-\nabla p, \\
& \frac{\partial \mathbf{b}}{\partial t}=\nabla \times(\mathbf{v} \times \mathbf{B})-\nabla \times \eta(\nabla \times \mathbf{b})
\end{aligned}
$$

with $\nabla \cdot \mathbf{b}=0$ and $\nabla \cdot \mathbf{v}=0$ (for incompressibility).

Using vector potentials for both the perturbed magnetic field and the velocity, these are written in the form:

$$
\begin{aligned}
& \frac{\partial(\nabla \times \nabla \times \mathbf{u})}{\partial t}=\nabla \times[(\nabla \times \mathbf{B}) \times(\nabla \times \mathbf{a})+(\nabla \times \nabla \times \mathbf{a}) \times \mathbf{B}], \\
& \frac{\partial \mathbf{a}}{\partial t}=(\nabla \times \mathbf{u}) \times \mathbf{B}+\nabla(\mathbf{B} . \mathbf{u})-\eta(\nabla \times \nabla \times \mathbf{a}),
\end{aligned}
$$

where $\mathbf{v}=\nabla \times \mathbf{u}$ and $\mathbf{b}=\nabla \times \mathbf{a}$, so that $\mathbf{u}$ is the velocity vector potential and $\mathbf{a}$ is the perturbed magnetic vector potential. The gauge is chosen so the the term $\nabla$ (B.u) is included in the second equation, which simplifies the numerical analysis of the second equation.

\section{Numerical analysis}

The equilibrium condition $\nabla P=\mathbf{J} \times \mathbf{B}$ is solved by using the 3D code VMEC under the assumption of nested flux surfaces. The solution was mapped to Boozer coordinates [9] with a Jacobian, $\mathcal{J} \propto B^{-2}$, to define a fluxcoordinate system $(s, \theta, \zeta)$.

The equilibrium magnetic field has the form

$$
\mathbf{B}=B^{\theta} \mathcal{J} \nabla \zeta \times \nabla s+B^{\zeta} \mathcal{J} \nabla s \times \nabla \theta=\frac{f(s)}{\mathcal{J}}(\mathcal{J} \nabla \zeta \times \nabla s+q(s) \mathcal{J} \nabla s \times \nabla \theta)
$$

which gives straight field lines with

$$
\text { B. } \nabla \propto \frac{\partial}{\partial \theta}+q(s) \frac{\partial}{\partial \zeta},
$$

where $q(s)$ is the safety factor. It is necessary to use Boozer coordinates to avoid singularities in the coordinate definition. The vector potentials are expressed in term of their covariant components, e.g.,

$$
\mathbf{a}=a_{s} \nabla s+a_{\theta} \nabla \theta+a_{\zeta} \nabla \zeta .
$$

These are expanded in a double Fourier series. A convenient representation is of the form

$$
a_{s}(s, \theta, \zeta, t)=\sum_{k=0}^{K} a_{s m(k) n(k)}(s) \exp [\mathrm{i} m(k) \theta-\mathrm{i} n(k) \zeta-\mathrm{i} \omega t],
$$

where $k$ labels the pairs of poloidal and toroidal mode numbers $m$ and $n$. 
Taking the double Fourier transform, the contravariant components of the Jacobian times the equation of motion

$$
-\mathrm{i} \omega \mathcal{J}(\nabla \times \nabla \times \mathbf{u})=\mathcal{J} \nabla \times[\mathbf{J} \times(\nabla \times \mathbf{a})+(\nabla \times \nabla \times \mathbf{a}) \times \mathbf{B}],
$$

become

$$
\begin{aligned}
-\mathrm{i} \omega \sum_{m} \sum_{n} \sum_{\beta}[ & R_{1 \alpha \beta}\left(m^{\prime}, m, n^{\prime}, n\right) u_{\beta m n}(s)+R_{2 \alpha \beta}\left(m^{\prime}, m, n^{\prime}, n\right) \frac{\partial u_{\beta m n}}{\partial s} \\
& \left.+\frac{\partial}{\partial s}\left(R_{3 \alpha \beta}\left(m^{\prime}, m, n^{\prime}, n\right) u_{\beta m n}\right)+\frac{\partial}{\partial s}\left(R_{4 \alpha \beta}\left(m^{\prime}, m, n^{\prime}, n\right) \frac{\partial u_{\beta m n}}{\partial s}\right)\right] \\
=\sum_{m} \sum_{n} \sum_{\beta}[ & P_{1 \alpha \beta}\left(m^{\prime}, m, n^{\prime}, n\right) u_{\beta m n}(s)+P_{2 \alpha \beta}\left(m^{\prime}, m, n^{\prime}, n\right) \frac{\partial a_{\beta m n}}{\partial s} \\
& \left.+\frac{\partial}{\partial s}\left(P_{3 \alpha \beta}\left(m^{\prime}, m, n^{\prime}, n\right) u_{\beta m n}\right)+\frac{\partial}{\partial s}\left(P_{4 \alpha \beta}\left(m^{\prime}, m, n^{\prime}, n\right) \frac{\partial a_{\beta m n}}{\partial s}\right)\right],
\end{aligned}
$$

where $\alpha$ and $\beta$ represent $(s, \theta, \zeta)$ and $R_{1 \alpha \beta}\left(m^{\prime}, m, n^{\prime}, n\right)$ etc. are double Fourier coefficients of the terms arising from the convolution. A similar structure arises from the Fourier decomposition of Ampere's law. In all a total of 105 functions have to be transformed to obtain the coefficients. Some saving was made by choosing the gauge so that the $\zeta$ component of $\mathbf{u}$ is zero and using only the $\theta$ and $\zeta$ components of the equation of motion above, taking account of the redundancy related to the fact that the divergence of this equation is zero. The algebra is challenging and involves a 3D non-orthogonal coordinate vector analysis. MATHEMATICA was used to do this analysis and to express the results in FORTRAN code to minimize coding errors.

It is expected that more than $60(m, n)$ components will generally be required to represent the modes. The resulting set of about 300 second-order differential equations gives a block-tri-diagonal matrix, after finite differencing in $s$. This treated the $s$-components on the half mesh points and the $\theta$-and $\zeta$-components on the full mesh arranged so that the divergence of a curl of any vector is exactly zero in the finite difference scheme used. This minimizes spurious modes. The growth rates are obtained in terms of the eigenvalues of this matrix. Typically a matrix involving about 300 sub-matrix blocks, each of size $300 \times 300$, is obtained. The eigenvalue analysis is done by inverse iteration. Because of the size of the problem the code was implemented on the VPP300 at the Australian National University using vector processing routines.
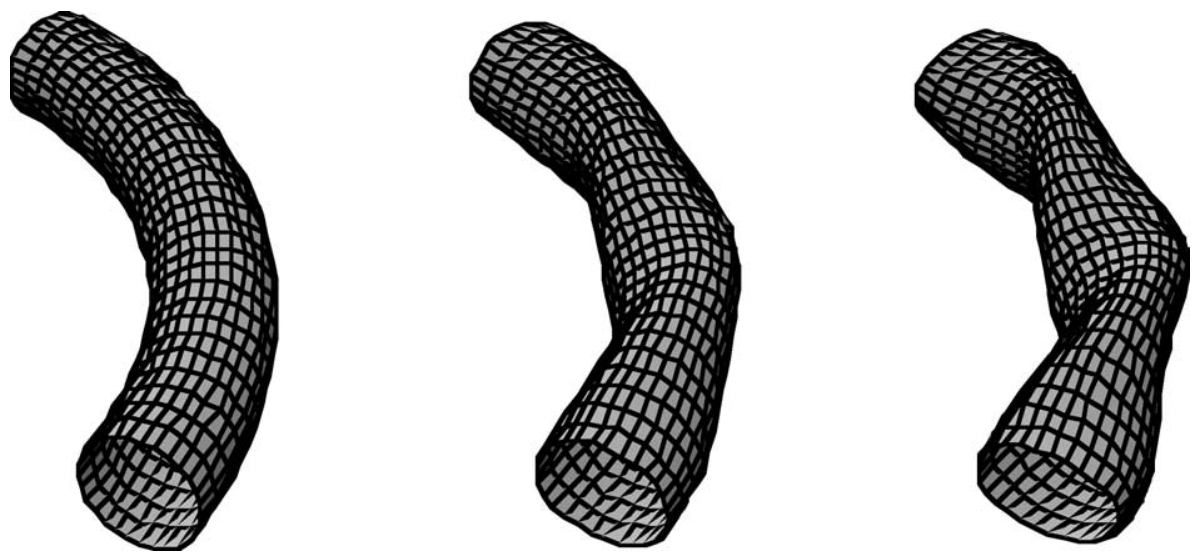

Fig. 1. Sequence with increasing helicity showing only two periods of a 10 period plasma outer surface. 


\section{Results}

The code has been tested in the axisymmetric limit by comparing against existing tokamak codes. We present here a limited number of preliminary results for model three-dimensional helical plasmas. Firstly, for a low-shear case whose axisymmetric limit is known we consider a sequence of plasmas with increasing helicity. Fig. 1 shows a sequence of plasma outer surfaces which are defined by the specification of the Fourier coefficients of the surface. In VMEC notation these correspond to plasmas with $R_{00}=10.0, Z_{00}=0.0, R_{10}=Z_{10}=1.0$ and the helicity $R_{11}=-Z_{11}$ taking the values 0.0,0.1 and 0.2. This is for the case (as in LHD [11]) where the number of field periods is equal to 10. Fig. 2 shows the ideal growth rate for a sequence like this as a function of increasing helicity.

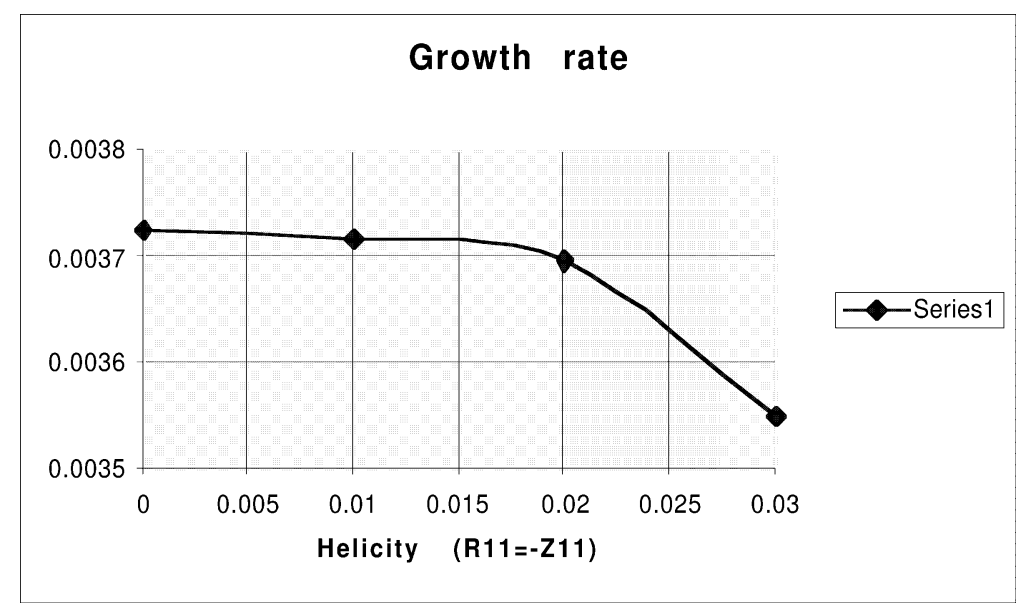

Fig. 2. Growth rate as a function of helicity.

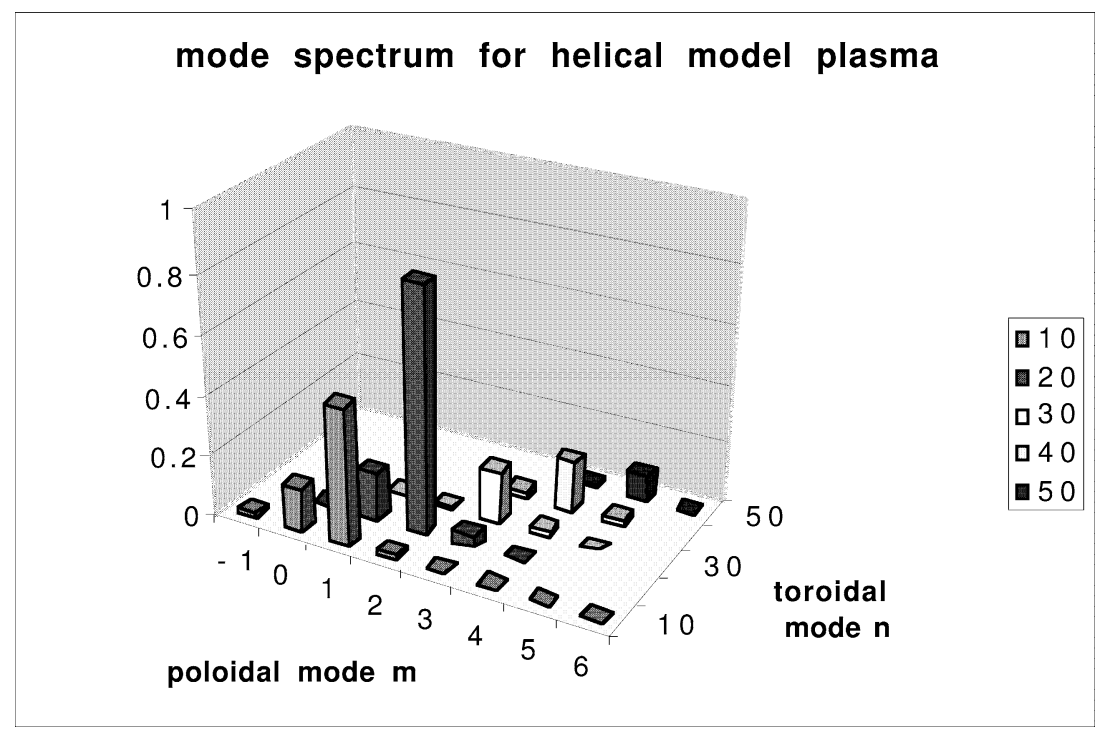

Fig. 3. Component strengths as a function of $m$ and $n$ for an unstable mode for a model helical plasma. 


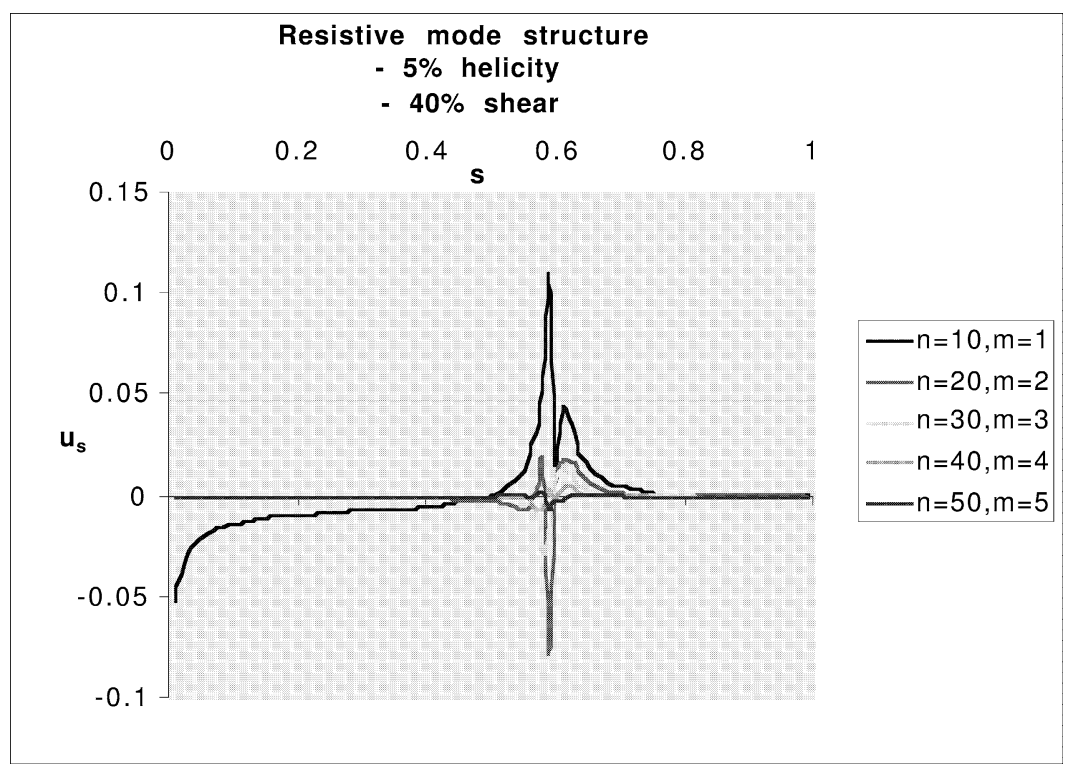

Fig. 4. Resistive mode structure for a model current-carrying plasma with helical twist.

It should be noted that when helicity is introduced there is coupling between all the components with different toroidal and poloidal mode numbers (not just poloidal mode coupling as in the axisymmetric case). Fig. 3 shows results from a typical unstable mode from this sequence, plotting a histogram of the integrated root-mean-square strengths of the components of a mode in terms of the mode numbers. In this case the dominant mode is the $n=20$, $m=2$ component but there are a number of other significant components too, particularly when $n / m=10$.

In tokamak plasmas it is known that the introduction of even a small amount of resistivity can give rise to a tearing mode, characterized by a sharply peaked eigenmode structure near a singular surface. Fig. 4 shows the mode structure of a helical tearing mode obtained by changing a tokamak-like (current-carrying) equilibrium with $40 \%$ shear by modifying the surface with $5 \%$ helicity (i.e. $R_{11}=-Z_{11}=0.05$ for the above sequence). This produces a tearing mode with coupled poloidal and toroidal components. This case is for a magnetic Reynolds number of $10^{7}$. These early results indicate the rich structure of the unstable modes which will be able to be obtained from the code.

\section{Acknowledgements}

This work has been supported by the Australian Research Grants Committee, the Australian Institute of Nuclear Science and Engineering and the Australian National University Supercomputer Centre. The authors are grateful to Dr S. Hirshman for the use of the VMEC code and Dr W.A. Cooper for the use of the section of TERPSICHORE which maps VMEC coordinates into Boozer coordinates.

\section{References}

[1] L.A. Charlton et al., J. Comp. Phys. 86 (1990) 270.

[2] A. Bondeson et al., Contr. Fusion Plasma Heating 14B (1990) 906.

[3] W. Kerner et al., J. Comp. Phys. 142 (1998) 271.

[4] M.H. Hughes, M.W. Phillips, R.G. Storer, Comput. Phys. Commun. 72 (1992) 76. 
[5] R.G. Storer, J. Comp. Phys. 66 (1986) 294.

[6] R.G. Storer, A.R. Schellhase, J. Comp. Phys. 123 (1996) 15.

[7] L. Garcia, Proc. EPS Conf. on Contr. Fusion and Plasma Physics, Praha, ECA 22C (1998) 1757.

[8] W.A. Cooper et al., Plasma Phys. Cont. Nuc. Fusion Res. 2 (1991) 793.

[9] C. Schwab, Phys. Fluids B 5 (1993) 3195.

[10] S.P. Hirshman, W.I. van Rij, P. Merkel, Comput. Phys. Commun. 43 (1986) 143.

[11] M. Fujiwara et al., J. Fusion Energy 15 (1996) 7. 